\title{
Supporting Information: \\ Pressure in Molecular Simulations with Scaled Charges. \\ 1. Ionic Systems
}

\author{
Jiří Kolafa \\ Department of Physical Chemistry, University of Chemistry and Technology, Prague, \\ Technická 5, 16628 Praha 6, Czech Republic \\ E-mail: jiri.kolafa@vscht.cz
}

\section{Contents}

S1 Electronic permittivity as a function of density via temperature dependence 2

S2 MDEC results with $\varepsilon_{\mathrm{f}}$ based on the underlying polarizable simulation instead of the Clausius-Mossotti formula $\quad 3$

$\begin{array}{ll}\text { S3 Isotherms for atomic ionic systems } & 7\end{array}$

S4 NaCl-like model crystal $\quad 8$

S4.1 Radial distribution functions $\ldots \ldots \ldots \ldots \ldots$

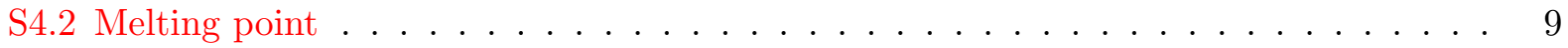

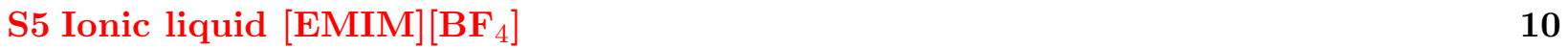

S5.1 Force field and the H5-F radial distribution functions . . . . . . . . . . . . . 10

S5.2 Isotherm . . . . . . . . . . . . . . . . . . . . . . 12 


\section{S1 Electronic permittivity as a function of density via tempera- ture dependence}

Data for the index of refraction for high-pressure ionic liquids are not available. As an indirect alternative, we show in Figure S1 the dependence of the electronic relative permittivity on density, where both quantities are functions of temperature at ambient pressure. In addition to 1-ethyl-3methylimidazolium tetrafluoroborate $\left([\mathrm{EMIM}]\left[\mathrm{BF}_{4}\right]\right.$, right), the same plot is shown for water for comparison (left).

At first glance, the Clausius-Mossotti (CM) equation with volume-independent polarizability (green lines) matches well the data for water [1]. The slope of the experimental $\varepsilon_{\mathrm{f}}(\rho)$ dependence for water close to $100{ }^{\circ} \mathrm{C}$ is $98 \%$ of the $\mathrm{CM}$ prediction, but it increases with decreasing temperature and diverges to infinity at water density maximum at $4{ }^{\circ} \mathrm{C}$. We can deduce that the slope ratio would be around 90-95\% if we could remove the anomalous part; this is close to the value of $\eta=0.88$ calculated for water at freezing point and increasing pressure (see Figure 1 of the paper). Therefore, parameter $\eta$ defined by eq 22 in the paper is likely only slightly smaller than the same parameter calculated indirectly using thermal expansion.

For $[\mathrm{EMIM}]\left[\mathrm{BF}_{4}\right]$, the slope ratio depends on the data set. Montalban et al. [2] data give $88 \%$, Shamsipur et al. [3] give 83\%, and Xu et al. [4] give $112 \%$. Note that the Xu et al. data differ considerably from the first two data sets; we thus suggest that the slope ratio is likely in the range 80-90 \% rather than greater than $100 \%$. Taking into account the above water value, we estimate the constant temperature slope ratio $\eta$ for $\left[\mathrm{EMIM}^{-}\left[\mathrm{BF}_{4}\right]\right.$ as $\eta=0.8 \pm 0.1$.
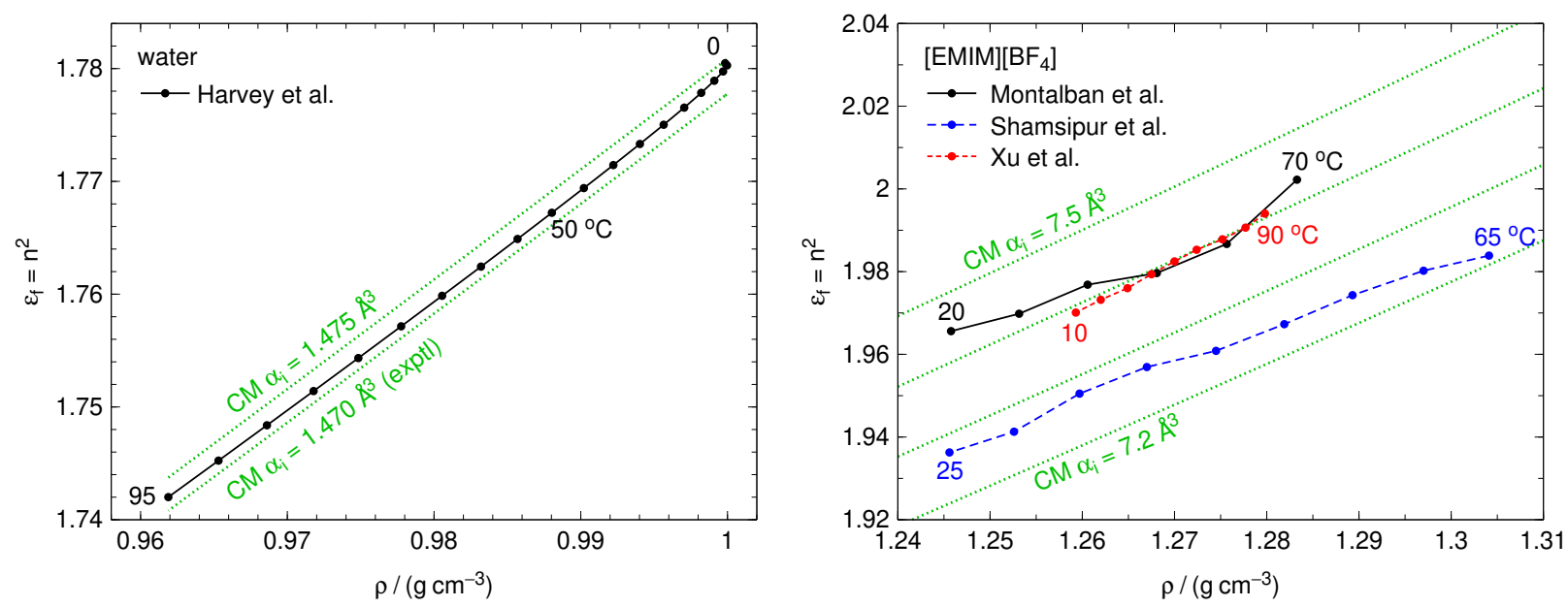

Figure S1: The electronic relative permittivity, $\varepsilon_{\mathrm{f}}=n^{2}$, as a function of density for water and $[\mathrm{EMIM}]\left[\mathrm{BF}_{4}\right]$ at ambient pressure. Numbers at points denote temperatures in ${ }^{\circ} \mathrm{C}$. 


\section{S2 MDEC results with $\varepsilon_{\mathrm{f}}$ based on the underlying polarizable sim- ulation instead of the Clausius-Mossotti formula}

For the sake of clarity and length, MDEC results with the value of the relative permittivity of the continuum $\left(\varepsilon_{\mathrm{f}}\right)$ calculated during the underlying polarizable simulation (MDEC/MD) are omitted in the paper and only the simulations with $\varepsilon_{\mathrm{f}}$ approximated by the Clausius-Mossotti (CM) equation are shown. In this section, richer versions of Tables 3 and 4 and Figure 2 are shown for completeness. Bulk moduli are not calculated for MDEC/MD because they would need the values of $\varepsilon_{\mathrm{f}}$ calculated in POL simulations for a series of densities. The differences between MDEC and MDEC/MD are small.

Table S1: Symmetric atomic ionic liquid simulated with polarizability (POL) and MDEC. In $\mathrm{MDEC} / \mathrm{MD}, \varepsilon_{\mathrm{f}}$ is calculated from polarizable simulation, otherwise the CM approximation applies. Symbols ECCR/ $\sigma$ and ECCR/ $\varepsilon$ denote that the LJ parameters were adjusted to best match the RDF of the original polarizable simulation. See the paper (Table 1) for the symbols of quantities. The standard statistical inaccuracies in the units of the least significant digit are at most 2 or are given in parentheses. Richer version of Table 3 in the paper.

\begin{tabular}{|c|c|c|c|c|c|}
\hline quantity & POL & MDEC/MD & MDEC & $\mathrm{ECCR} / \sigma$ & $\mathrm{ECCR} / \varepsilon$ \\
\hline$\alpha$ & 2.5 & 0 & 0 & 0 & 0 \\
\hline$\sigma_{\mathrm{LJ}}$ & 3.405 & 3.405 & 3.405 & 3.23165 & 3.405 \\
\hline$\epsilon_{\mathrm{LJ}}$ & 119.8 & 119.8 & 119.8 & 119.8 & 62.4 \\
\hline$\varepsilon_{\mathrm{f}}$ & 1 & $1.9769(4)$ & 1.9308 & 1.9308 & 1.9308 \\
\hline$U$ & -335 & -312 & -313 & -319 & -315 \\
\hline$U_{1}$ & 21 & 9 & 9 & 1 & 11 \\
\hline$U_{2}$ & -372 & -164 & -169 & -170 & -169 \\
\hline$U_{3}$ & 16 & -156 & -153 & -156 & -156 \\
\hline$U_{\mathrm{el}}$ & -356 & -321 & -321 & -326 & -326 \\
\hline$\tilde{U}$ & & -156 & -160 & -162 & -158 \\
\hline$U_{\text {pol }}$ & -335 & -328 & -328 & -327 & -327 \\
\hline$P$ & 374 & -580 & -550 & -924 & -779 \\
\hline$P_{1}$ & 5435 & 3332 & 3360 & 2925 & 3076 \\
\hline$P_{2}$ & & 1987 & 1891 & 2050 & 2044 \\
\hline$P_{3}$ & & -5899 & -5801 & -5899 & -5899 \\
\hline$P_{\mathrm{el}}$ & -5059 & -3912 & -3910 & -3849 & -3855 \\
\hline$\tilde{P}_{2}$ & & -2058 & -2112 & -2124 & -2118 \\
\hline$P_{2}^{\text {corr }}$ & & 4045 & 4003 & 4174 & 4162 \\
\hline$P_{\text {vir }}$ & & -685 & -663 & -1158 & -1001 \\
\hline$P_{3}^{\text {corr }}$ & & -3940 & -3890 & -3940 & -3940 \\
\hline$\tilde{P}$ & & 1274 & 1248 & 801 & 958 \\
\hline$P_{\mathrm{pol}}$ & 376 & -1299 & -1275 & 630 & 450 \\
\hline$B$ & $2597(14)$ & & $769(2)$ & & \\
\hline$\tilde{B}$ & & & $5315(2)$ & & \\
\hline$\kappa(\mathrm{EMD})$ & $253(9)$ & $339(11)$ & $338(12)$ & & \\
\hline$\kappa(\mathrm{NEMD})$ & $260(3)$ & $340(6)$ & $341(4)$ & & \\
\hline$D_{\text {ion }}$ & $2.200(5)$ & $2.337(7)$ & $2.307(7)$ & & \\
\hline
\end{tabular}


Table S2: Five pairs of ions (charges $\pm 0.5 e$ ) in 290 atoms of "polarizable argon" with column MDEC/MD added. See the paper (Table 1) for the symbols of quantities. Richer version of Table 4 in the paper.

\begin{tabular}{cccc}
\hline quantity & POL & MDEC/MD & MDEC \\
\hline$\alpha$ & 2.5 & 0 & 0 \\
$\varepsilon_{\mathrm{f}}$ & 1 & $1.9548(2)$ & 1.9308 \\
\hline$U$ & 1.52 & 1.46 & 1.47 \\
$U_{1}$ & 3.67 & 3.60 & 3.60 \\
$U_{2}$ & -2.42 & -0.61 & -0.62 \\
$U_{3}$ & 0.52 & -1.29 & -1.27 \\
$U_{\mathrm{el}}$ & -1.91 & -1.90 & -1.89 \\
$\tilde{U}$ & & 2.75 & 2.74 \\
$U_{\text {pol }}$ & 1.52 & 1.53 & 1.52 \\
\hline$P$ & 2385 & 2375 & 2375 \\
$P_{1}$ & 2429 & 2416 & 2417 \\
$P_{2}$ & & 7 & 7 \\
$P_{3}$ & & -49 & -48 \\
$P_{\text {el }}$ & -43 & -42 & -41 \\
$\tilde{P}_{2}$ & & -8 & -8 \\
$P_{2}^{\text {corr }}$ & & 15 & 15 \\
$P_{\text {vir }}$ & & 2393 & 2393 \\
$P_{3}^{\text {corr }}$ & & -33 & -32 \\
$\tilde{P}$ & & 2409 & 2409 \\
$P_{\text {pol }}$ & 2385 & 2374 & 2375 \\
\hline$B$ & $6276(9)$ & & $6267(2)$ \\
$\tilde{B}$ & & & $6331(2)$ \\
$\kappa($ EMD $)$ & $5.30(17)$ & $5.59(18)$ & $5.74(19)$ \\
$\kappa(\mathrm{NEMD})$ & $5.02(25)$ & $5.80(25)$ & $5.96(25)$ \\
$D_{\text {ion }}$ & $3.85(6)$ & $3.88(6)$ & $3.88(6)$ \\
\hline & & &
\end{tabular}


Table S3: Ionic NaCl-like crystal. Richer version of Table 5 in the paper.

\begin{tabular}{cccc}
\hline quantity & POL & MDEC/MD & MDEC \\
\hline$\alpha$ & 1.5 & 0 & 0 \\
$\varepsilon_{\mathrm{f}}$ & 1 & 1.7649 & 1.7627 \\
\hline$U$ & -369 & -355 & -355 \\
$U_{1}$ & 16 & 13 & 13 \\
$U_{2}$ & -387 & -213 & -214 \\
$U_{3}$ & 2 & -154 & -154 \\
$U_{\mathrm{el}}$ & -385 & -368 & -368 \\
$\tilde{U}$ & & -201 & -201 \\
$U_{\mathrm{pol}}$ & -369 & -366 & -366 \\
\hline$P$ & 13 & 0 & 2 \\
$P_{1}$ & 6989 & 6238 & 6240 \\
$P_{2}$ & & 2406 & 2396 \\
$P_{3}$ & & -8644 & -8634 \\
$P_{\mathrm{el}}$ & -6977 & -6238 & -6238 \\
$\tilde{P}_{2}$ & & -3809 & -3814 \\
$P_{2}^{\text {corr }}$ & & 6216 & 6210 \\
$P_{\text {vir }}$ & & -330 & -329 \\
$P_{3}^{\text {corr }}$ & & -5885 & -5879 \\
$\tilde{P}$ & & 2428 & 2425 \\
$P_{\text {pol }}$ & 12 & -652 & -651 \\
\hline$B$ & $16722(15)$ & & $13574(70)$ \\
$\tilde{B}$ & & & $19699(60)$ \\
$T_{\mathrm{melt}}$ & $1235(10)$ & & \\
\hline & & &
\end{tabular}



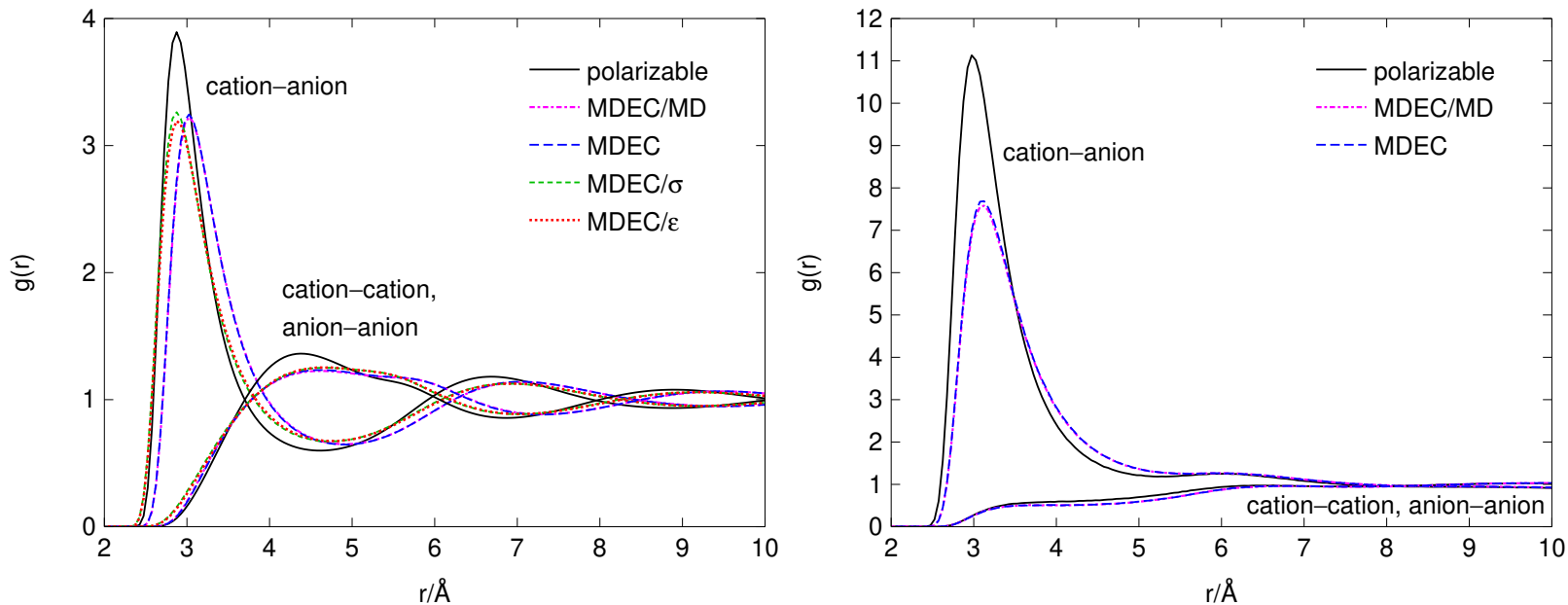

Figure S2: Radial distribution functions for systems of Tables S1 (ionic liquid, left) and S2 (ionic solution, right) with MDEC/MD lines added. Richer version of Figure 2 in the paper. 


\section{S3 Isotherms for atomic ionic systems}

Figure S3 shows the isotherms $(T=2000 \mathrm{~K})$ for the model ionic systems of Tables S1 and S2. The data (points) were fitted to quartic polynomials. From these polynomials, the bulk moduli at density of $1.5 \mathrm{~g} / \mathrm{cm}^{3}$ were calculated as well as the equilibrium densities needed to calculate the errors in densities.
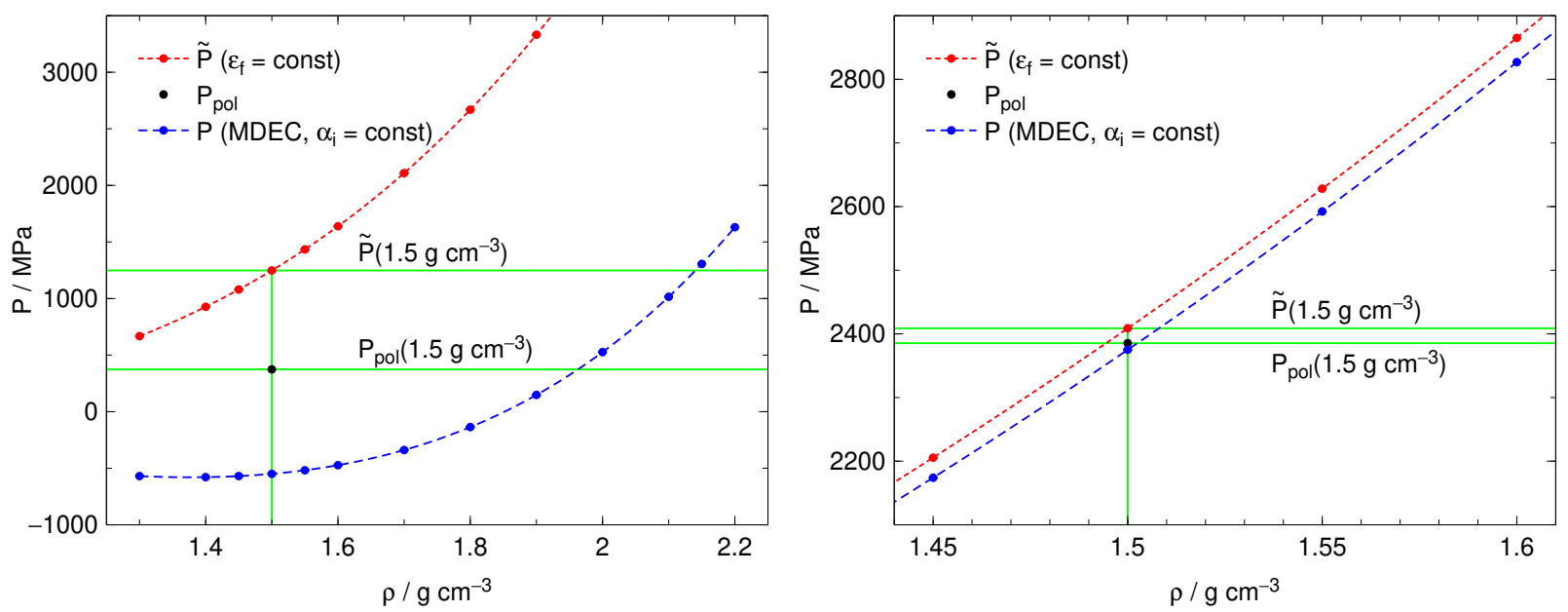

Figure S3: Pressure as the function of density for model symmetric ionic liquid (left) and solution of 5 ion pairs in "polarizable argon" (right). $\tilde{P}$ is the conventional uncorrected MDEC result (i.e., with $\varepsilon_{\mathrm{f}}$ independent on density), $P_{\mathrm{pol}}$ is obtained by the equivalent polarizable simulation, and $P$ is the MDEC value with density-independent $\alpha_{i}$. The reference density is emphasizes by green lines. 


\section{S4 NaCl-like model crystal}

\section{S4.1 Radial distribution functions}

The radial distribution function cation-anion of a NaCl-like symmetric crystal in Figure S4 shows that the first peak of the polarizable version is slightly shifted left. Although the symmetry of the rock-salt structure is not broken, the stronger cation-anion attraction in the POL version is responsible for this small shift. MDEC (with CM-based $\varepsilon_{\mathrm{f}}$ ) and MDEC/MD $\left(\varepsilon_{\mathrm{f}}\right.$ determined from a POL simulation) are indistinguishable even in the zoomed inset.

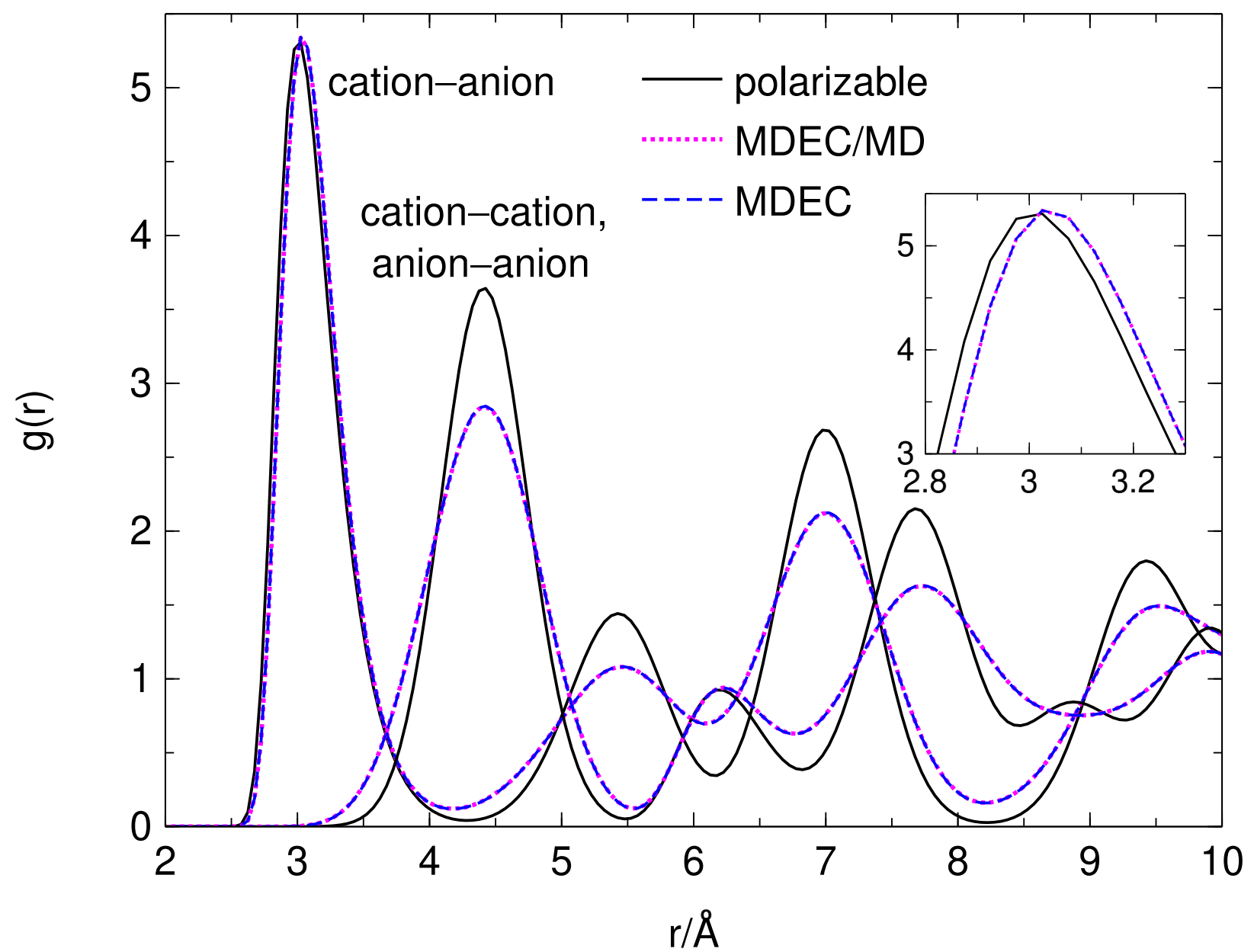

Figure S4: Radial distribution functions for the NaCl-like crystal of Table S3 (Table 5 in the paper). 


\section{S4.2 Melting point}

The melting point of the polarizable model of the crystal was determined in the slab geometry $[5$, $6,7,8]$. First, a crystal was simulated at pressure $0.1 \mathrm{MPa}$ at given temperature to determine the equilibrium box size. This box was then replicated three times and a bit stretched in the $z$-direction. Half the box was melted using a position-based thermostat. Then, a barostat in the $z$-direction was applied with $x, y$-sizes kept constant. From the melting/freezing curves (Figure S6), the following melting points were determined: $1259(5) \mathrm{K}$ for $N=3 \times 216$ and $1245(4) \mathrm{K}$ for $N=3 \times 512$. The $1 / N$-extrapolation gives the melting temperature of $1235(10) \mathrm{K}$.

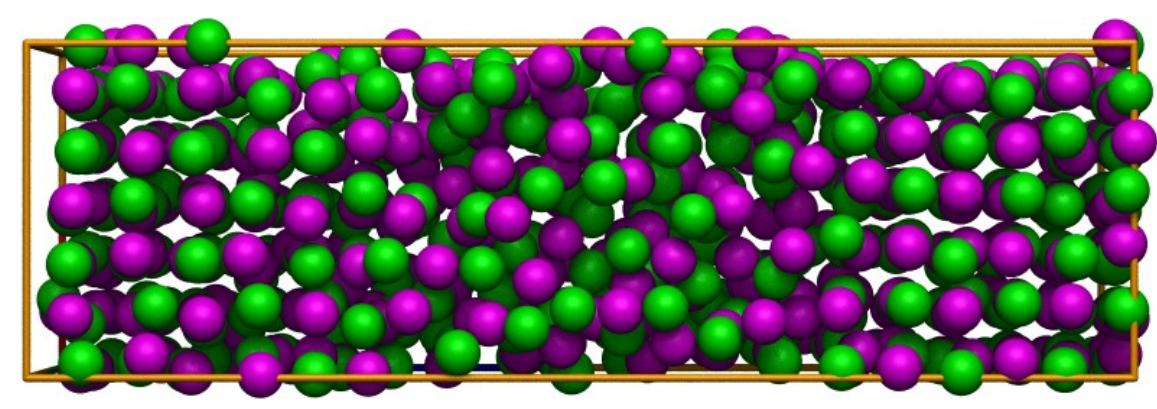

Figure S5: The slab geometry, $N=3 \times 216$.
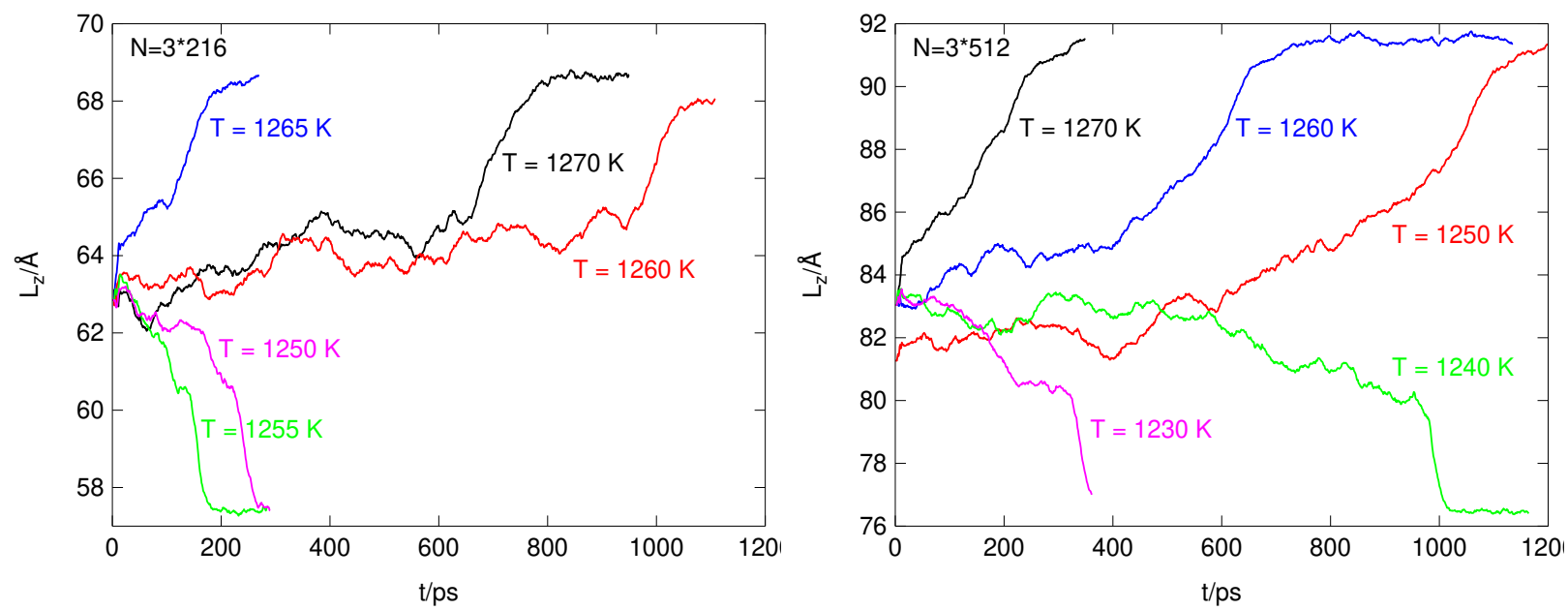

Figure S6: Melting curves of slabs of a crystal in equilibrium with liquid. 


\section{S5 Ionic liquid [EMIM] $\left[\mathrm{BF}_{4}\right]$}

\section{S5.1 Force field and the H5-F radial distribution functions}

The force field for 1-ethyl-3-methylimidazolium tetrafluoroborate ([EMIM] $\left.\left[\mathrm{BF}_{4}\right]\right)$ was taken from ref. [9]. Atom numbering, partial charges and polarizabilities are shown in Figure S7. The radial distribution function of hydrogen $\mathrm{H} 5$ and fluorine is shown in Figure $\mathrm{S} 8$ for density $1284 \mathrm{~kg} / \mathrm{m}^{3}$ except our NPT simulation which gives by about $1 \%$ higher density. It is seen that our results are in a mediocre but still acceptable agreement with the original paper, but digitized results from later work[10] show a bad agreement for the H5-F radial distribution function.

In paper [10], charge scaling of 0.814 is proposed. The disagreement of our MDEC results for the H5-F radial distribution function persists, see Figure S9 . Our NPT simulation gives density lower by about $1 \%$ than reported[10].
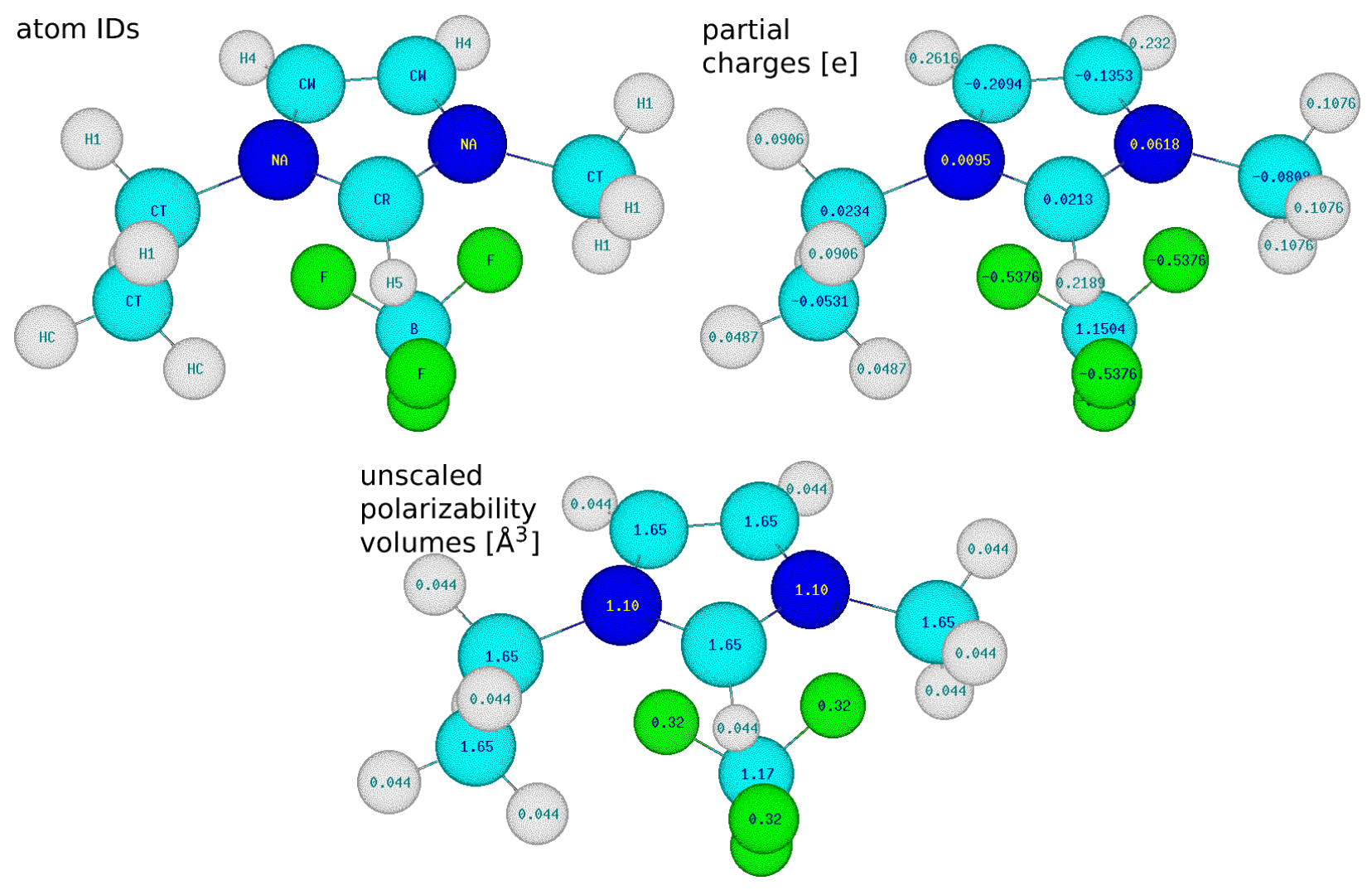

Figure S7: Atom names and partial charges in the $[\mathrm{EMIM}]\left[\mathrm{BF}_{4}\right]$ force field [9] and assigned polarizabilities [11] before scaling by 0.605022 . 


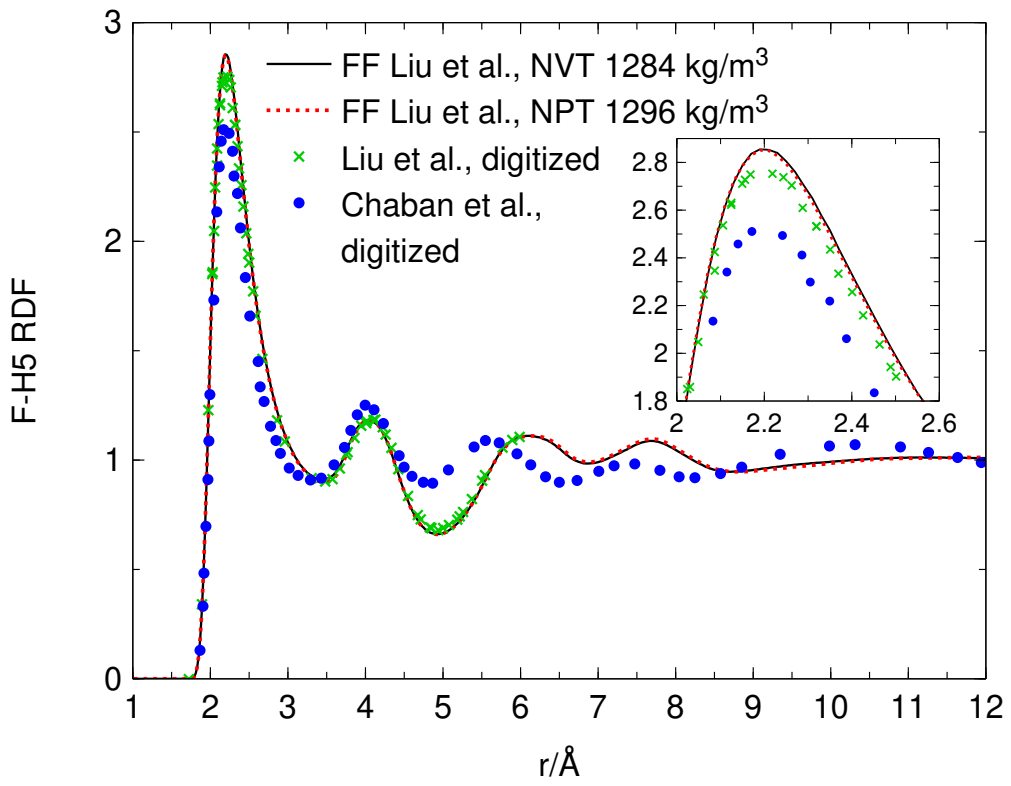

Figure S8: The F-H5 radial distribution function for the force field by Liu et al. [9], calculated in this work and compared to the digitized figures[9, 10].

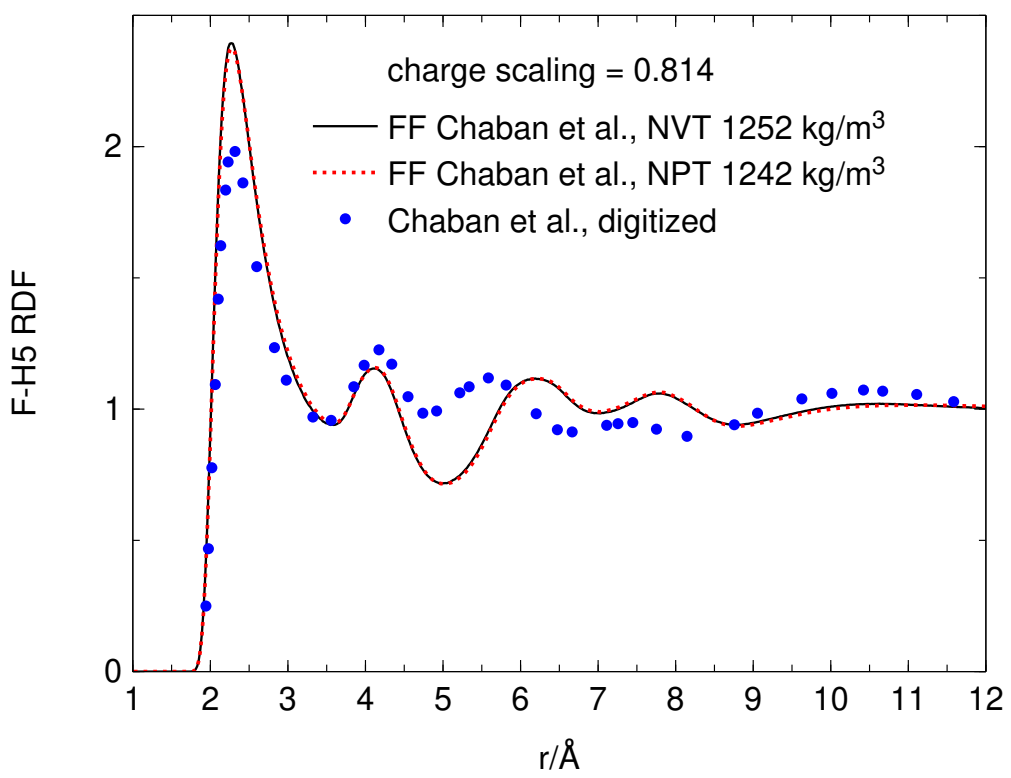

Figure S9: The F-H5 radial distribution function for the Liu et al.[9] force field with charges scaled by $0.814[10]$ calculated in this work and compared to the digitized figure[10]. 


\section{S5.2 Isotherm}

In figure S10, the calculations with different densities are provided. The error bars are smaller than symbol sizes.

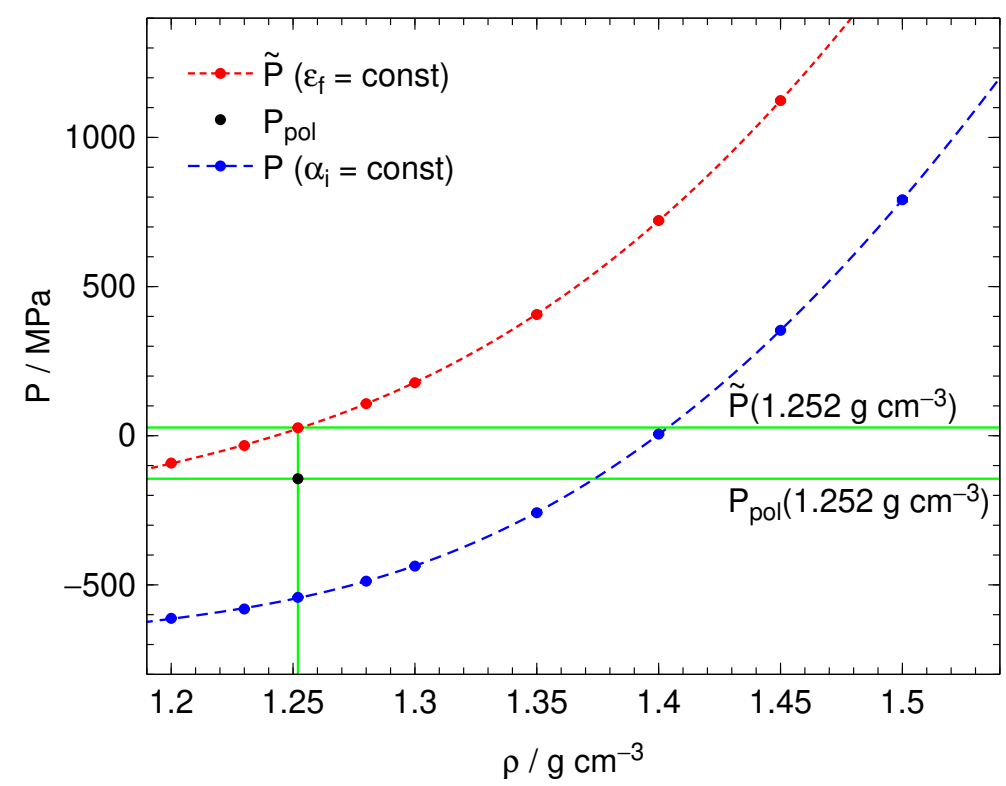

Figure S10: Pressure as the function of density for $[\mathrm{EMIM}]\left[\mathrm{BF}_{4}\right] . \tilde{P}$ is the conventional uncorrected MDEC result (i.e., with $\varepsilon_{\mathrm{f}}$ independent on density), $P_{\mathrm{pol}}$ is obtained by the equivalent polarizable simulation, and $P$ is the MDEC value with density-independent $\alpha_{i}$. The reference density is emphasizes by green lines. 


\section{References}

[1] A. Harvey, J. Gallagher, J. Sengers, Revised formulation for the refractive index of water and steam as a function of wavelength, temperature and density, J. Phys. Chem. Ref. Data 27 (4) (1998) 761-774. doi:\{10.1063/1.556029\}.

[2] M. G. Montalban, C. L. Bolivar, F. G. Diaz Banos, G. Villora, Effect of temperature, anion, and alkyl chain length on the density and refractive index of 1-alkyl-3-methylimidazolium-based ionic liquids, J. Chem. Eng. Data 60 (7) (2015) 1986-1996. doi:\{10.1021/je501091q\}.

[3] M. Shamsipur, A. A. M. Beigi, M. Teymouri, S. M. Pourmortazavi, M. Irandoust, Physical and electrochemical properties of ionic liquids 1-ethyl-3-methylimidazolium tetrafluoroborate, 1-butyl-3-methylimidazolium trifluoromethanesulfonate and 1-butyl-1-methylpyrrolidinium bis(trifluoromethylsulfonyl)imide, J. Mol. Liq. 157 (1) (2010) 43-50. doi:\{10.1016/j .molliq. $2010.08 .005\}$.

[4] W.-G. Xu, L. Li, X.-X. Ma, J. Wei, W.-B. Duan, W. Guan, J.-Z. Yang, Density, surface tension, and refractive index of ionic liquids homologue of 1-alkyl-3-methylimidazolium tetrafluoroborate $\left[\mathrm{C}_{n} \operatorname{mim}\right]\left[\mathrm{BF}_{4}\right](n=2,3,4,5,6)$, J. Chem. Eng. Data 57 (8) (2012) 2177-2184. doi: $\{10.1021 /$ je3000348\}.

[5] A. J. C. Ladd, L. Woodcock, Triple-point coexistence properties of the Lennard-Jones system, Chem. Phys. Lett. 51 (1977) 155-159. doi:10.1016/0009-2614(77)85375-X.

[6] J. L. Aragones, E. Sanz, C. Valeriani, C. Vega, Calculation of the melting point of alkali halides by means of computer simulations, J. Chem. Phys. 137 (2012) 104507. doi:10.1063/ 1.4745205 .

[7] J. Anwar, D. Frenkel, M. G. Noro, Calculation of the melting point of $\mathrm{NaCl}$ by molecular simulation, J. Chem. Phys. 118 (2003) 728-735. doi:10.1063/1.1522375.

[8] J. Kolafa, Solubility of $\mathrm{NaCl}$ in water and its melting point by molecular dynamics in the slab geometry and a new BK3-compatible force field, J. Chem. Phys. 145 (20) (2016) 204509. doi:10.1063/1.4968045.

[9] Z. Liu, S. Huang, W. Wang, A refined force field for molecular simulation of imidazolium-based ionic liquids, J. Phys. Chem. B 108 (34) (2004) 12978-12989. doi:\{10.1021/jp048369o\}.

[10] V. Chaban, Polarizability versus mobility: atomistic force field for ionic liquids, Phys. Chem. Chem. Phys. 13 (35) (2011) 16055-16062. doi:\{10.1039/c1cp21379b\}.

[11] QUANTA, Parameter Handbook Release 3.0, Polygen Corporation, 1990. 